\title{
A SHORT PROOF OF A THEOREM OF BARR-BECK
}

BY Y.-C. WU

Communicated by David A. Buchsbaum, June 9, 1970

Let $\mathbf{C}$ be a category. Let $(P, \mathfrak{T})$ be a projective structure $[\mathrm{K}-\mathrm{W}]$ where $P$ are the set of $刃$-projectives and the set of $P$-proper morphisms. Then the following are true.

I. $(P, \mathfrak{T})$ is determined by a cotriple iff there is a coreflexive subcategory $C^{\prime} \subset C$ with the properties:

(1) $\left|\mathbf{C}^{\prime}\right| \subset P$,

(2) the coreflexions are in $\Re$.

II. If $S \dashv T$, where $S: C \rightarrow D$ and $T: D \rightarrow C$, and if $(P, \mathfrak{T C})$ is a projective structure in $C$ determined by a cotriple $G$, then the projective structure $\left(r S P, T^{-1} \mathfrak{T}\right)$ is determined by the cotriple $S G T$, where $r S P$ is the collection of retracts of $S P$. Moreover, if $(P, \mathfrak{N})$ is induced by a cotriple $G$, then $\left(r S P, T^{-1} \mathfrak{T}\right)$ is induced by $S G T$.

The proofs of these two statements are omitted here. As a corollary of the above statements, we have the following.

III (Barr-Beck). The triple cohomology of groups coincides with the Eilenberg MacLane cohomology.

IV (Barr-Beck). The triple cohomology of associative algebras coincides with the Hochschild cohomology.

For detailed statements of the above, see $\left[B-B_{1}\right]$.

We now prove III. Let $(G, \pi)$ be the category of groups over the group $\pi$. Let $M$ be a $\pi$-module. Then there is an adjoint pair

$$
(G, \pi) \underset{T}{\stackrel{S}{\rightleftarrows}} \pi \text {-Mod }
$$

where $S(W)=Z \pi \otimes_{W} I W$ with $I W=\operatorname{ker}(Z(W) \rightarrow Z)$ and $T(M)$ $=M \times_{\varphi} \pi$, the semidirect product of $M$ and $\pi$ with respect to the $\pi$ module structure $\varphi: \pi \rightarrow \operatorname{Aut}(M)$ (cf. $\left[\mathrm{B}-\mathrm{B}_{2}\right]$, where $S(W)$ is denoted by $\left.\operatorname{Diff}_{x}(W)\right)$. Now the free group cotriple on the category $G$ of groups gives a cotriple on $(G, \pi)$. Let $(P, \Re)$ be the corresponding projective structure. Then $\left(r S P, T^{-1} \mathfrak{T}\right)$ is a projective structure in $\pi$-Mod. To show $\left(r S P, T^{-1} \mathfrak{T}\right)$ is induced by the free functor cotriple on $\pi$-Mod, it suffices to show that $S P$ contains all free $\pi$-modules. Since $P$ are retracts of free groups and $I F$ are free

AMS 1969 subject classifications. Primary 0830, 1310.

Key words and phrases. Category, functors, cotriples, projective structures, cohomology groups. 
$F$-modules (cf. [M, p. 123]), $S(F)$ are indeed free $\pi$-modules. Now by adjointness, we have

$$
\left[G W, M \times_{\varphi} \pi\right] \cong[S G W, M]
$$

where $G$ is the free cotriple on $G$. We can conclude that $S G W$ is homotopic to the bar resolution of $S(W)$ by direct computation or by invoking the following:

V. Let $A$ be a preadditive category. Let $G$ be a cotriple on $A$ where the functor $G$ is additive. Then the cotriple complex of every $B \in A$ is a projective resolution.

The statement IV can be proved similarly with the pair of functors,

$$
(K \text {-alg, } \wedge) \underset{T}{\stackrel{S}{\rightleftarrows}} \wedge^{e} \text {-Mod }
$$

with $S(\Gamma)=J \Gamma \otimes_{\Gamma} e \wedge^{e}$, where $J \Gamma=\operatorname{ker}\left(\wedge \otimes_{K} \wedge^{\mathrm{opp}} \rightarrow \wedge\right)$, and $T(M)=\wedge^{e} * M[\mathrm{~B}]$. The proof goes after we observe that $J \Gamma$ is a free $\Gamma^{e}$-module if $\Gamma$ is free $K$-algebra, [C-E, p. 181].

AdDEd IN Proof. Another way to prove III and IV is to observe that if $T$ preserves and reflects epimorphisms, then the projective structure $\left({ }_{\gamma} S P, T^{-1} \mathscr{N}\right)$ is an absolute projective structure if $(P, \mathfrak{T})$ is.

\section{REFERENCES}

[B] J. Beck, Thesis, Columbia University, New York, 1967.

[K-W] H. Kleisli and Y.-C. Wu, On injective sheaves, Canad. Math. Bull. 7 (1964), 415-423. MR 29 \#5882.

[E-M] S. Eilenberg and J. C. Moore, Adjoint functors and triples, Illinois J. Math. 9 (1965), 381-398. MR 32 \#2455.

[B- $\left.\mathrm{B}_{1}\right]$ M. Barr and J. Beck, Acyclic models and triples, Proc. Conference Categorical Algebra (La Jolla, Calif., 1965) Springer, New York, 1966, pp. 336-343. MR 39 \#6955.

[M] S. MacLane, Homology, Die Grundlehren der math. Wissenschaften, Band 114, Academic Press, New York; Springer-Verlag, Berlin, 1963. MR 28 \#122.

[C-E] H. Cartan and S. Eilenberg, Homological algebra, Princeton Univ. Press, Princeton, N. J., 1956. MR 17, 1040.

$\left[\mathrm{B}-\mathrm{B}_{2}\right] \mathrm{M}$. Barr and J. Beck, Homology and standard constructions, Lecture Notes in Math., no. 80, Springer-Verlag, Berlin and New York.

Oakiand University, Rochester, Michigan 48063 